\title{
Design of Low-Complexity Hybrid Precoder and Inkjet-Printed Antenna Array for Massive MIMO Downlink Systems
}

\author{
Subitha D $(D)$ and Mathana J M \\ S.A. Engineering College, Anna University, Department of Electronics and Communication Engineering, Chennai, India \\ Correspondence should be addressed to Subitha D; subithab@saec.ac.in
}

Received 25 August 2017; Revised 15 January 2018; Accepted 31 January 2018; Published 24 April 2018

Academic Editor: Maggie Y. Chen

Copyright (c) 2018 Subitha D and Mathana J M. This is an open access article distributed under the Creative Commons Attribution License, which permits unrestricted use, distribution, and reproduction in any medium, provided the original work is properly cited.

\begin{abstract}
The dramatically growing mobile communication industry necessitates the demand for the speedy and error-free connectivity at considerably low cost for the billions of users. This is made possible only through the technological advancements that replace the current $4 \mathrm{G}$ wireless systems by $5 \mathrm{G}$. Massive MIMO is the key technology used in $5 \mathrm{G}$ that offers spectral efficiency of up to 3 times and throughput of up to 10 times the current 4G. The additional antennas used in massive MIMO systems help in many ways but lack in complexity. Hence, in this paper, we propose two design methodologies to reduce the complexity of massive MIMO systems. The first one is the design of low-complexity hybrid precoder based on Zero-Forcing (ZF) precoding algorithm and Neumann series approximation. The second one is the design of flexible, environment friendly, simple 128-element antenna array at the frequency of $2.4 \mathrm{GHz}$ using inkjet printing technology. The substrate used for printing is the "glossy paper" with dielectric constant of 2.31, and the ink used is silver nanoparticle ink with conductivity of 35,700,000 s/m. The element used for the formation of array is the z-shaped coplanar waveguide (CPW) monopole antenna. The performance of the proposed designs is evaluated in terms of probability of error for the hybrid precoding algorithm and radiation characteristics like gain, directivity, and return loss for the printed antenna design.
\end{abstract}

\section{Introduction}

The future of wireless networks is in the hands of technologies that provide good quality, intense security, and improved reliability. The most promising $5 \mathrm{G}$ wireless standards are expected to satisfy all the above requirements by providing higher data rate, low latency between radio links, and minimal power consumption than the existing network. Though the above-mentioned performance targets do not need to be met simultaneously, they provide the basis for the Gbits/s user experience for $5 \mathrm{G}$ networks [1-3].

The large-scale (massive) MIMO is one of the promising technologies that can meet the above performance targets in which a central node (base station) with antennas in the order of 100's serves multiple users (mobile terminals) with single or small number of antennas [4-7]. When the number of base station (BS) antennas $M$ grows very large or tends to infinity, the effects of noise and fast fading present in the channel vanish [8]. Increasing $M$ also makes the diagonal elements of the Gram matrix $\left(\mathbf{G}=\mathbf{H} \mathbf{H}^{\mathrm{H}}\right)$ more dominant for the given channel matrix $H$. This implies that the channels of the independent users become orthogonal which completely eliminates the interuser interference. But for practical systems with $M \ll \infty$, the Inter-user interference can be mitigated by applying precoding techniques at the transmitter side. But the major problem associated with this system is its complexity due to the large dimensional matrices that changes frequently at the rate proportional to the channel's Doppler spread. Hence, in this paper, we propose two design approaches to simplify the complexity issue: the first one is the low-complexity hybrid precoding algorithm and the second one is the design of antenna array using simple and cheap inkjet printing technology.

The first part of this paper is the design of low-complexity hybrid precoding algorithm for massive MIMO systems. Among various precoding algorithms available, the hybrid precoding has gained more attention in the recent times due to its complexity and cost trade-offs [9]. The drawback of pure digital precoding is the requirement of dedicated RF modules for each of the BS antennas. Since the array size of 
the massive MIMO system is typically large, digital precoding becomes more complex in terms of area, cost, and power limitations. But the hybrid precoder architecture shown in Figure 1 groups the transmitting antenna elements into a number of subarray modules [10]. Hence, separate RF chains are assigned for each subarray module rather than individual antenna element which reduces the cost of the system considerably. Hybrid precoder combines a low-dimensional baseband precoder (digital) with a high-dimensional RF precoder (analog). The digital precoder controls the signal supplied to the RF precoder by the complex weight matrix $\mathbf{W}_{\mathrm{D}}$, and the complex analog weight matrix $\mathbf{W}_{\mathrm{A}}$ of the $\mathrm{RF}$ precoder controls the phase shift network of the antenna array. More extensive research works have been carried out on developing hybrid precoding algorithms in [9-12]. All these works devise various hybrid algorithms for massive antenna systems based on computationally intensive block diagonalization and eigenvalue decomposition methods. Though plenty of works evolved to reduce the complexity of these methods, their implementation in hardware is not feasible as they deal with lot of division operations. Since the hardware like FPGAs are more friendly with MAC (multiply and accumulate) operations, we propose here a hardware friendly zero forcing (ZF) algorithm-based [13] hybrid precoder. Though ZF algorithm is simple, the matrix inversion associated with this algorithm is highly expensive as the number of user antennas increases. Thus, the complexity of the $\mathrm{ZF}$ algorithm is greatly reduced by replacing large-scale matrix inversion by low-complexity Neumann series-based approximation [14].

The second part of the work introduces a simple, low-cost, environment friendly antenna design for massive MIMO systems using inkjet printing technology [15]. The performance of the design is verified using CST MWS tool. The ink used for printing is silver nanoparticle ink, and substrate used for printing is the flexible $2 \mathrm{D}$ "glossy paper."

The rest of this paper is organized as follows. Section 2 describes the low-complexity hybrid precoding algorithm and its performance characteristics simulated using MATLAB. Then, the antenna design using inkjet printing technology and its performance using CST MWS is discussed in Section 3.

\section{Low-Complexity Hybrid Precoder Design}

2.1. System Description. Consider the downlink massive MIMO system where a base station (BS) with $M$ transmit antennas communicates with $K$ mobile users equipped with single receiving antenna. The modulated data vector $\mathbf{m}$ intended for $K$ users is represented as

$$
\mathbf{m}=\left[\begin{array}{llll}
m_{1} & m_{2} & \ldots & m_{K}
\end{array}\right]^{\mathrm{T}} \in \mathbb{C}^{K \times 1} .
$$

This modulated data vector is first processed by $N \times K$ digital precoder $\mathbf{W}_{\mathrm{D}}$ followed by $N$ number of RF chains. Here, we critically assume that the proposed precoder architecture requires at least $K$ number of $\mathrm{RF}$ chains
$(N=K)$ to enable multistream data transmission of $K$ users. The output of the digital precoder is carried over by the respective RF chains and converted into analog domain. Next, they are processed by $M \times N R F$ precoder $\mathbf{W}_{\mathrm{A}}$ that controls the phase angle of the phase shifters connected to each of the antenna elements. Thus, the $M \times 1$ precoded data vector is expressed as [16]

$$
\mathbf{s}=\mathbf{W} \mathbf{m}=\mathbf{W}_{\mathrm{A}} \mathbf{W}_{\mathrm{D}} \mathbf{m},
$$

where $\mathbf{s}=\left[\begin{array}{llll}s_{1} & s_{2} & \ldots & s_{M}\end{array}\right]^{\mathrm{T}} \in \mathbb{C}^{M \times 1}$. In (2), the data vector $\mathbf{m}$ is transformed into the symbol vector $\mathbf{s}$ by means of the linear precoder matrix $\mathbf{W}$. Here, the weight matrix $\mathbf{W}$ of size $M \times K$ is split into two as $\mathbf{W}_{\mathrm{D}}$ and $\mathbf{W}_{\mathrm{A}}$. This precoded data vector $\mathbf{s}$ is then transmitted over the MIMO channel $\mathbf{H}$ that yields

$$
\mathbf{y}=\mathbf{H s}+\mathbf{n},
$$

where $\mathbf{y} \in \mathbb{C}^{K \times 1}$ is the data vector received by $K$ single antenna users, $\mathbf{n}$ is the white Gaussian noise of unit variance, zero mean, and $\mathbf{H}=\left[\begin{array}{llll}\mathbf{H}_{1}^{\mathrm{T}} & \mathbf{H}_{2}^{\mathrm{T}} & \ldots & \mathbf{H}_{K}^{\mathrm{T}}\end{array}\right]^{\mathrm{T}} \in \mathbb{C}^{K \times M}$ where $\mathbf{H}_{k} \in \mathbb{C}^{1 \times M}$ is the channel gain of the $k$ th user with single antenna. If each user is equipped with $N_{\mathrm{U}}$ number of antennas rather than single, the channel gain of each user is given by $\mathbf{H}_{k} \in \mathbb{C}^{N_{\mathrm{U}} \times M}$. Thus, the received signal at the $k$ th user terminal is given by [17]

$$
\mathbf{y}_{k}=\mathbf{H}_{k} \mathbf{W}_{k} \mathbf{m}_{k}+\sum_{i=1, i \neq k}^{K} \mathbf{H}_{i} \mathbf{W}_{i} \mathbf{m}_{i}+\mathbf{n} .
$$

The first term in above expression is the desired signal of the intended user, the second term is the interference from other users, and the last term $\mathbf{n}$ is the noise added by the channel. As massive MIMO significantly reduces the effects of channel noise and fast fading effects, the interference due to other users (interuser interference) need to be taken care. Zero forcing $(\mathrm{ZF})$ is one technique of linear precoding in which the precoding matrix is the optimal solution obtained by forcing the interuser interference in each of the user terminals to zero. In (4), by forcing the second term that denotes the interuser interference to zero, we obtain the solution for $\mathbf{W}$ as given below [17].

$$
\mathbf{W}=\mathbf{H}^{\mathrm{H}}\left(\mathbf{H} \mathbf{H}^{\mathrm{H}}\right)^{-1}
$$

The above equation can now be represented using Gram matrix $\mathbf{G}$ as

$$
\mathbf{W}=\mathbf{H}^{\mathrm{H}} \mathbf{G}^{-1},
$$

where Gram matrix $\mathbf{G}=\mathbf{H H}^{\mathrm{H}} \in \mathbb{C}^{K \times K}$ and substituting the above equation of $\mathbf{W}$ in (2) we get the precoded data vector

$$
\mathbf{s}=\mathbf{H}^{\mathrm{H}} \mathbf{G}^{-1} \mathbf{m} .
$$




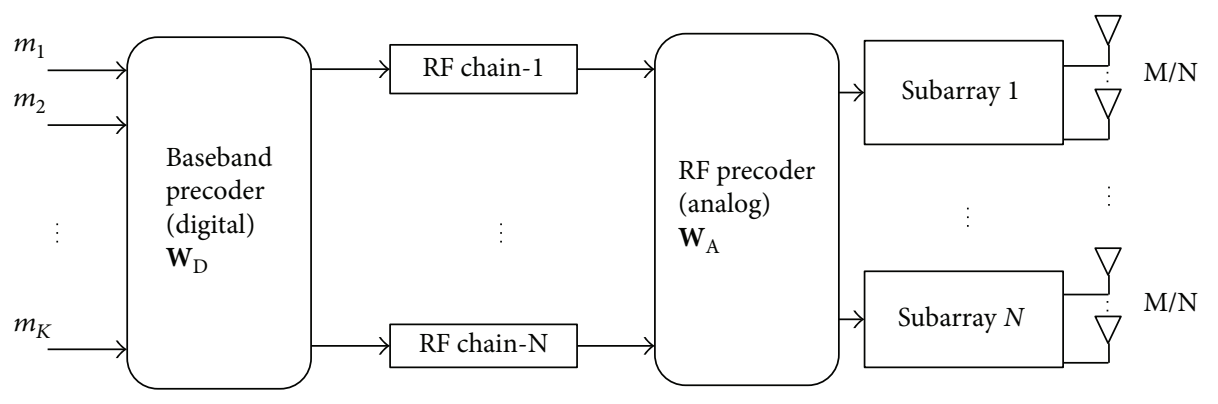

FIGURE 1: Block diagram of the hybrid precoder structure.

To obtain the precoded data vector s given in (7), the steps involved in the ZF precoder are Gram matrix computation $\mathbf{G}$, inversion of Gram matrix $\mathbf{G}^{-1}$, multiplication of $\mathbf{G}^{-1}$ with $\mathbf{m}$ and $\mathbf{H}^{\mathrm{H}}$. While implementing the above structure in hardware, the Gram matrix computation module and multiplication module requires less complex tasks since they need only multiplier modules that can easily be implemented in hardware MAC units. But the inverse computation module requires division operation that is highly infeasible in hardware. Hence, finding $\mathbf{G}^{-1}$ is computationally challenging, especially, when the dimension of the matrix is quite high [8]. Thus, we aim to reduce the complexity of the ZF precoding algorithm by replacing direct inversion by simple mathematical approximations such as Neumann series.

2.2. Low-Complexity Hybrid Precoding Algorithm. According to [14], $\mathbf{G}^{-1}$ in (7) is approximated using Neumann series as

$$
\mathbf{G}^{-1} \approx \sum_{n=0}^{L}\left(\mathbf{I}-\mathbf{Z}^{-1} \mathbf{G}\right)^{n} \mathbf{Z}^{-1}
$$

where $\mathbf{I}$ is the identity matrix of size $K$ and matrix $\mathbf{Z}$ is an initial approximation of $\boldsymbol{G}$, mostly it is chosen as the diagonal matrix containing the diagonal elements of $\mathbf{G}$ and hence much easier to invert. Computing (8) requires $L-1$ matrix multiplications where $L$ is the order of the polynomial. Here, (8) reaches equality as $L$ tends to infinity and the convergence rate is initiated by the correct selection of Z. Using the above stated approximation, the RF precoder matrix $\mathbf{W}_{\mathrm{A}}$ is obtained first with the knowledge of channel matrix $\mathbf{H}$ as given below

$$
\mathbf{W}_{\mathrm{A}}=\mathbf{H}^{\mathrm{H}} \mathbf{G}^{-1} \approx \mathbf{H}^{\mathrm{H}} \sum_{n=0}^{L}\left(\mathbf{I}-\mathbf{Z}^{-1} \mathbf{G}\right)^{n} \mathbf{Z}^{-1}
$$

Once the $\mathrm{RF}$ precoder $\mathrm{W}_{\mathrm{A}}$ is found, we can readily find the digital precoding matrix $\mathbf{W}_{\mathrm{D}}$ from the $\mathrm{ZF}$ algorithm given in (5) as

$$
\mathbf{W}_{\mathrm{D}}=\mathbf{H}_{\mathrm{d}}^{\mathrm{H}}\left(\mathbf{H}_{\mathrm{d}} \mathbf{H}_{\mathrm{d}}^{\mathrm{H}}\right)^{-1}
$$

where $\mathbf{H}_{\mathrm{d}}$ is the effective channel gain of the digital precoder by assuming fixed analog precoder. Hence, the effective

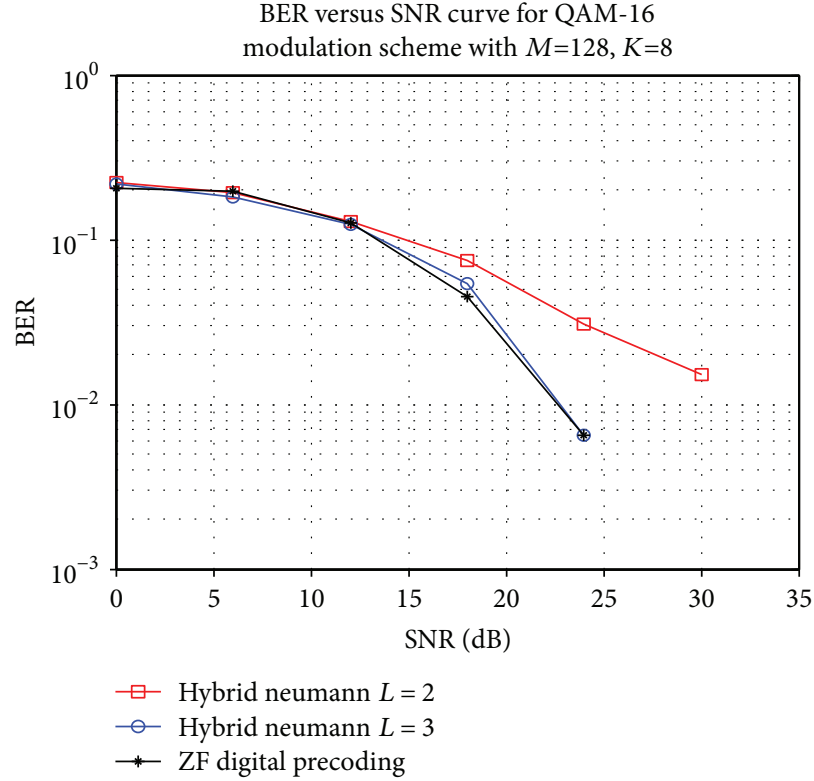

FIGURE 2: BER performance of hybrid precoding in urban micro cell scenario for $128 \times 8$ massive MIMO system.

channel including the analog precoder $\mathbf{W}_{\mathrm{A}}$ is given as $\mathbf{H}_{\mathrm{d}}=$ $\mathbf{H W}_{\mathrm{A}}$. Then, substituting in (10) yields

$$
\mathbf{W}_{\mathrm{D}}=\left(\mathbf{H} \mathbf{W}_{\mathrm{A}}\right)^{\mathrm{H}}\left(\mathbf{H} \mathbf{W}_{\mathrm{A}}\left(\mathbf{H} \mathbf{W}_{\mathrm{A}}\right)^{\mathrm{H}}\right)^{-1} \text {. }
$$

As we know that $\left(\mathbf{H W}_{\mathrm{A}}\right)^{\mathrm{H}}=\mathbf{W}_{\mathrm{A}}{ }^{\mathrm{H}} \mathbf{H}^{\mathrm{H}}$ (conjugate transpose property) the above equation becomes

$$
\mathbf{W}_{\mathrm{D}}=\mathbf{W}_{\mathrm{A}}{ }^{\mathrm{H}} \mathbf{H}^{\mathrm{H}}\left(\mathbf{H} \mathbf{W}_{\mathrm{A}} \mathbf{W}_{\mathrm{A}}{ }^{\mathrm{H}} \mathbf{H}^{\mathrm{H}}\right)^{-1} \text {. }
$$

The inversion operation in (12) is also approximated using Neumann approximation. Finally, the RF and digital precoders can be designed using the relations (10) and (12), respectively, for the hybrid precoder architecture.

The proposed algorithm which is based on ZF algorithm can also be extended for the downlink system with users equipped with $N_{\mathrm{U}}$ number of antennas instead of single antenna [17]. This yields the complex channel matrix of size $K N_{\mathrm{U}} \times M$ which in turn expands the corresponding precoder matrix size by an amount of $N_{U}$. Hence, the demand for the low-complexity algorithms is higher as the size of the matrix grows. 


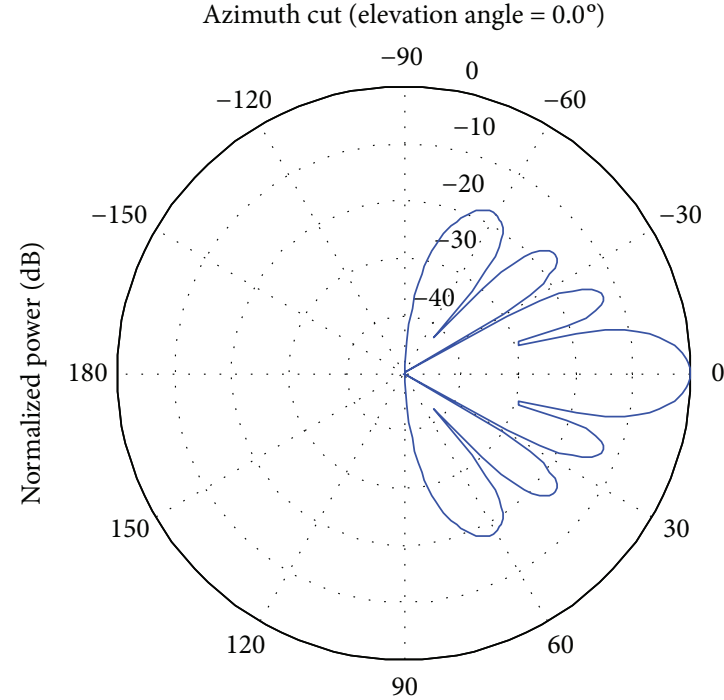

Normalized power $(\mathrm{dB})$, broadside at 0.00 degrees

(a) 128-element array

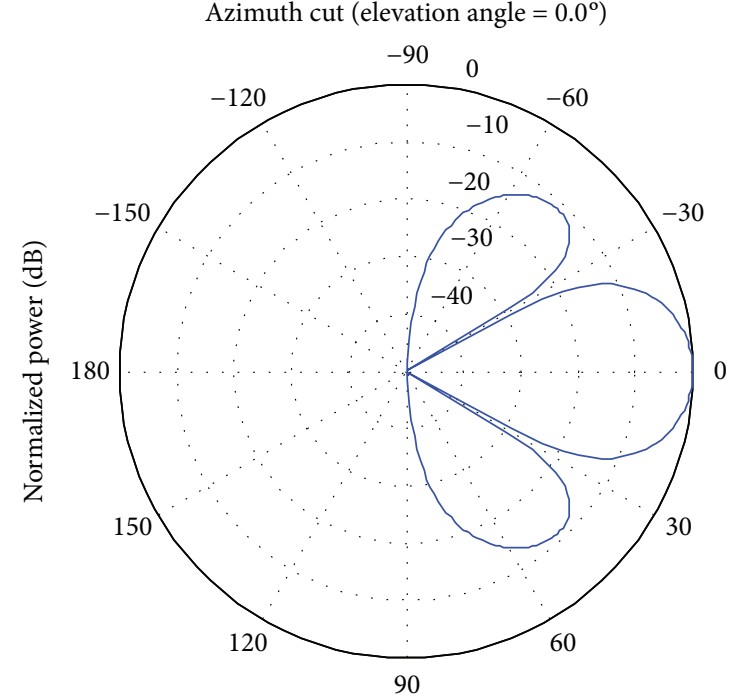

Normalized power $(\mathrm{dB})$, broadside at 0.00 degrees

(b) 64-element array

FIGURE 3: Radiation pattern of the antenna array with 16 subarrays in rectangular geometry.

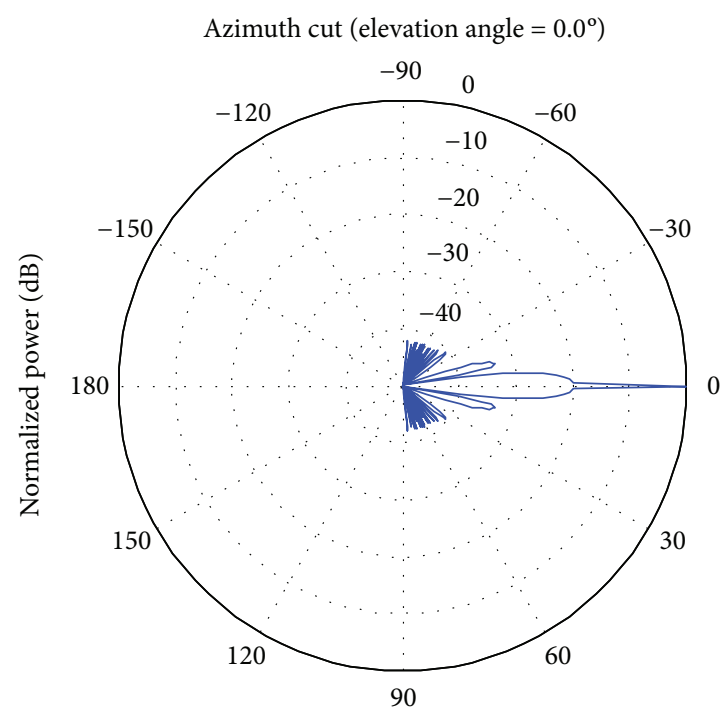

Normalized power $(\mathrm{dB})$, broadside at 0.00 degrees

(a) 128-element array

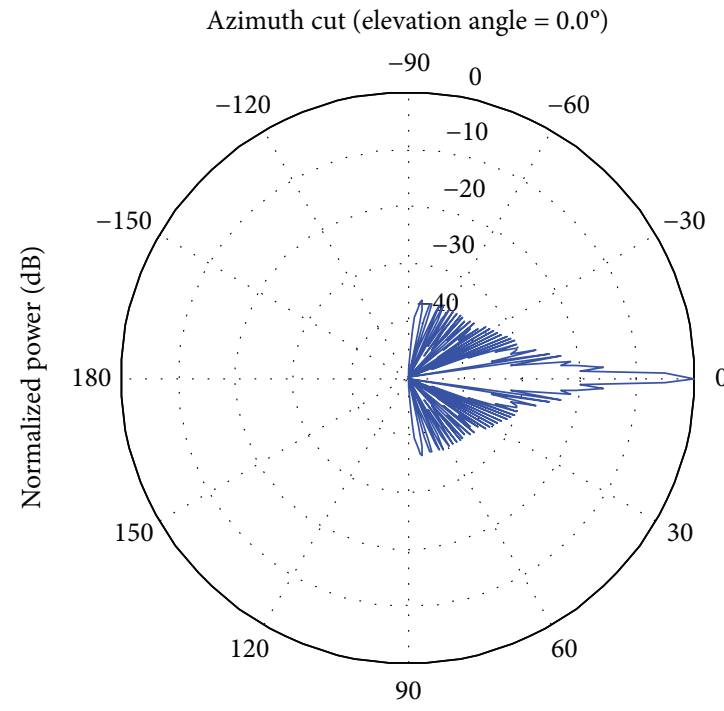

Normalized power $(\mathrm{dB})$, broadside at 0.00 degrees

(b) 64-element array

FIgURE 4: Radiation pattern of the ULA with 16 subarrays.

2.3. Simulation Results. This subsection illustrates the BER performance of the proposed Neumann seriesbased hybrid precoding algorithm, and comparisons are made with direct inversion-based digital precoding. The performance is evaluated for a massive MIMO downlink system with BS equipped with $M=128$ uniform linear antenna array with antenna spacing of $d=\lambda / 2$ serving $K=8$ mobile users. The above system requires the knowledge of downlink channel matrix $\mathbf{H}$ of size $K \times M$ for the computation of precoding matrices $\mathbf{W}_{\mathrm{A}}$ and $\mathbf{W}_{\mathrm{D}}$. This complex channel matrix is simulated using WINNER phase-II [18] model.
TABle 1: Properties of ink and substrate used for printing.

\begin{tabular}{lc}
\hline Ink properties & \\
Viscosity & $2.3 \mathrm{mPa} \cdot \mathrm{sec}$ \\
Surface tension & $35 \mathrm{mN} / \mathrm{m}$ \\
Conductivity & $35,700,000 \mathrm{~S} / \mathrm{m}$ \\
\hline "Glossy paper" substrate properties \\
Relative permittivity & 4.01 \\
Loss tangent & 0.07 \\
Dielectric constant & 2.31 \\
Thickness & $0.1 \mathrm{~mm}$ \\
\hline
\end{tabular}




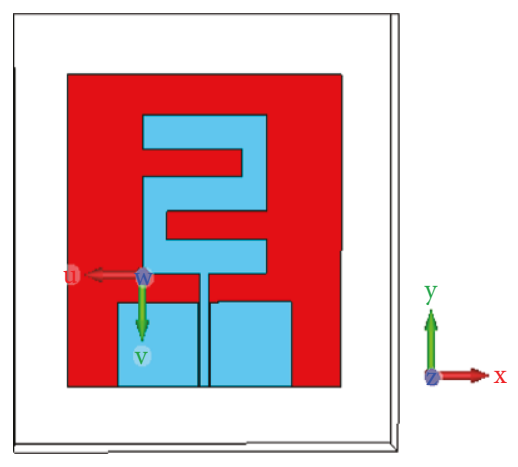

(a)

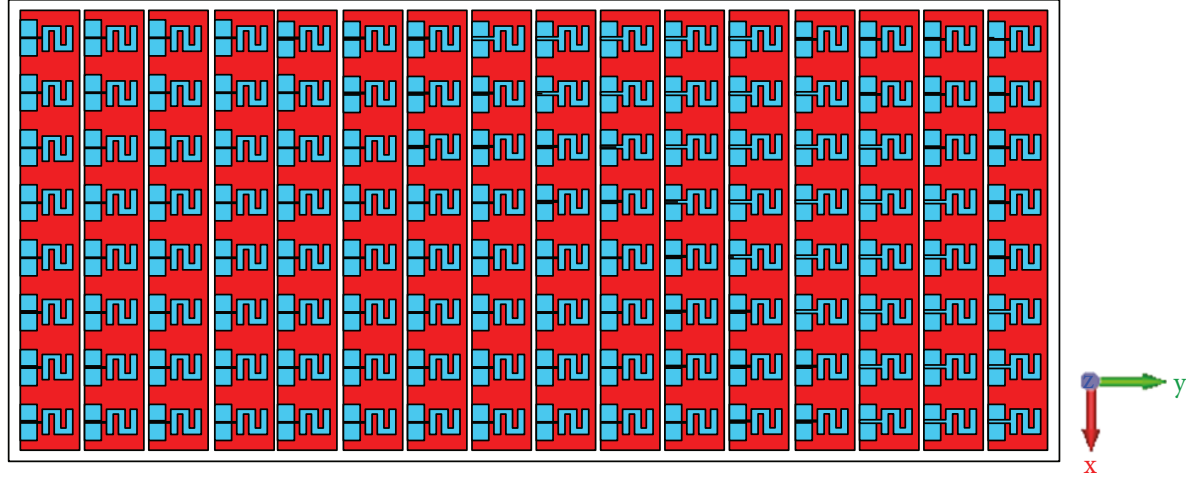

(b)

Figure 5: (a) Single Z-shaped CPW monopole antenna. (b) Array of 128 Z-shaped CPW monopole elements.

The simulations are carried out for a propagation scenario of urban micro cell where both BS and MSs are surrounded by uniform distribution of scatterers located in an elliptical region covering the entire street accommodating both BS and mobile stations (MS). The coverage area of BS is chosen as 500 meters with the antenna heights well below the surrounding buildings. Data sequences are generated randomly and mapped using 16-QAM to obtain $\mathbf{m}$. The modulated symbols are multiplied with $\mathbf{W}_{\mathrm{A}}$ and $\mathbf{W}_{\mathrm{D}}$ to get $\mathbf{s}$. Then, $\mathbf{s}$ is transmitted through the channel where it is added with zero mean, unit variance Gaussian noise to obtain $\mathbf{y}$. The error performance is calculated for the system described above, and Figure 2 shows the performance of Neumann-based hybrid precoding for order $L=1$ and 2 . The simulation results clearly exhibit that the error performance reaches the optimal value of ZF precoding (direct inversion based) as $L$ increases.

\section{Massive MIMO Antenna Design}

3.1. Background Knowledge of Antenna Array. Considering the points discussed above to facilitate the hybrid precoder architecture for massive MIMO system, it is required to divide the antenna elements into subarray modules. Each of these subarray modules is fed by dedicated RF chains, thus reducing the number of $\mathrm{RF}$ chains required by individual antenna element. The geometry of array and the number of subarray elements have a major impact on the radiation characteristics of the system. For massive MIMO systems to support multiuser transmission, multiple beams are needed to focus on each user. The results simulated in MATLAB shown below depicts the radiation pattern of 64 and 128 antenna arrays with 16 subarray modules where each subarray comprises 4 and 8 elements, respectively. From the results, it is evident that the rectangular array provides multibeam radiation characteristics with the number of beams almost equal to the number of elements in the subarray. The higher the number of elements in the subarray, the larger is the number of beams. Thus, the array geometry and number of subarray elements clearly decide on the radiation pattern of the massive antenna array. From Figures 3 and 4 , it is clear that the uniform linear array (ULA) geometry of subarrays produces many ripples with no considerable main beams, as in the case of rectangular geometry, which produces multiple beams with reasonable gain. Hence, to support multibeam transmission of $5 \mathrm{G}$ wireless applications, rectangular geometry of subarrays is preferred over linear pattern.

3.2. Printed Antenna Array Design-Simulation Results. Next, we focus on the design of 128-element massive antenna array in a more effective, flexible way using inkjet printing technology. The operating frequency of the proposed design is $2.4 \mathrm{GHz}$ as this lies in the frequency range used for the typical mobile radio communication worldwide. The substrate used for printing is the cheap, readily available, environmental friendly material "glossy paper." The major advantage of this material is its flexibility to various applications. The ink used for printing is the conductive silver nanoparticle ink having conductivity of $35,700,000 \mathrm{~S} / \mathrm{m}$. The properties of the ink and paper substrate are given in Table 1.

Figure 5(a) shows the design Z-shaped monopole element with the dimensions given in [15], and Figure 5(b) depicts the 128-element massive array. This arrangement is having 16 subarrays with each subarray integrated with 8 Z-shaped elements. The subarray spacing of the proposed design is $10 \mathrm{~mm}$. Figures $6(\mathrm{a})$ and $6(\mathrm{~b})$ plot the return loss characteristics over the frequency range of $2-6 \mathrm{GHz}$ for the antenna array and single element, respectively. It is seen that the $S_{1,1}$ value is considerably less than $14 \mathrm{~dB}$ over the frequency range of interest in both plots. The notch at $2.4 \mathrm{GHz}$ is clearly visible in both plots. The graphs shown in Figures $7(\mathrm{a})$ and $7(\mathrm{~b})$ are the $2 \mathrm{D}$ model of the gain radiation pattern at phi $=0$ and 90 degrees, respectively. Figures $8(\mathrm{a})$ and $8(\mathrm{~b})$ depict $3 \mathrm{D}$ model of gain and directivity pattern. From all these, it is clear that the proposed design exhibits a reasonably high gain of around $27.3 \mathrm{~dB}$ at the frequency of $2.4 \mathrm{GHz}$ with the beamwidth of 7.5 degrees at $\mathrm{phi}=0$ degrees. This proposed design outperforms the recent microstrip massive antenna array design printed on LTCC substrate whose gain is just $7 \mathrm{~dB}$ [19]. Thus, the proposed inkjet-printed antenna array produces nearly $20 \mathrm{~dB}$ gain improvement over the previous work. 


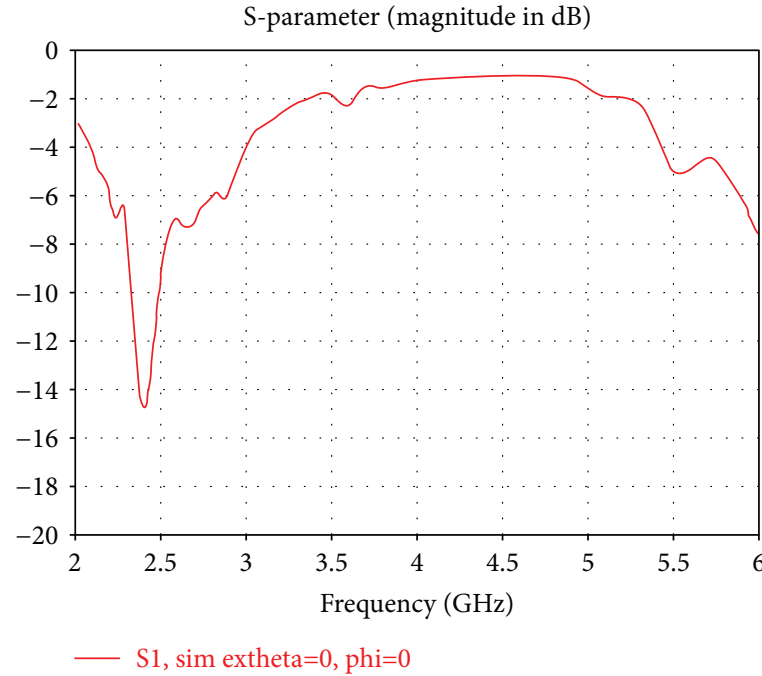

(a)

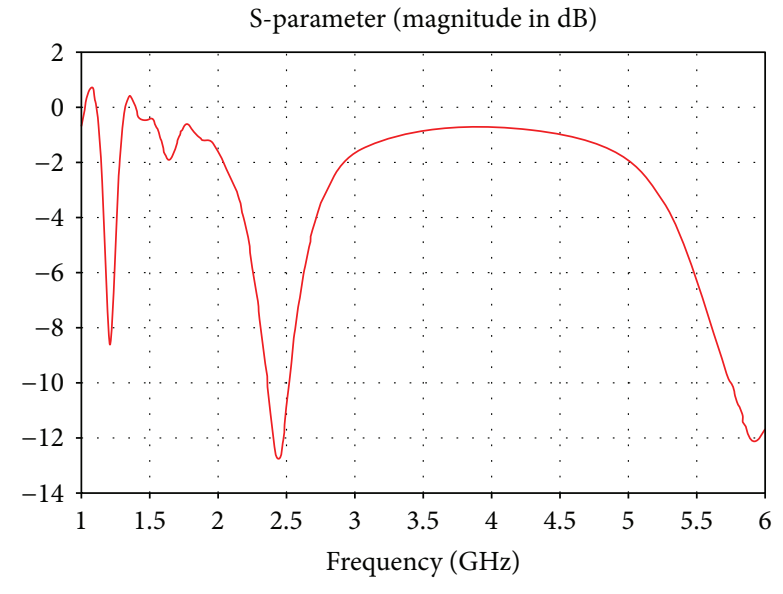

(b)

FIGURE 6: (a) S-parameter graph of 128-element array. (b) S-parameter graph of single Z-shaped element.

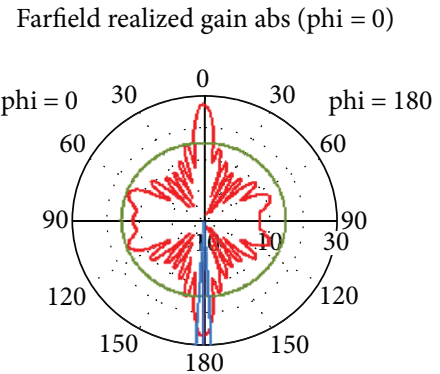

Theta/degree versus $\mathrm{dB}$

Farfield realized gain abs $(\mathrm{phi}=90)$

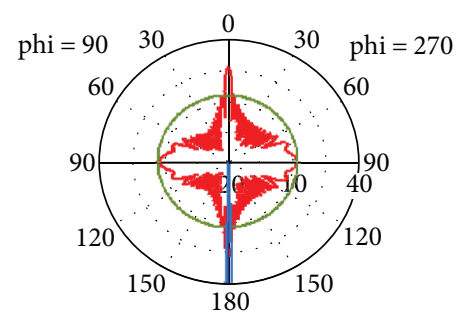

Theta/degree versus $\mathrm{dB}$
— Farfield $(f=2.4)$ [SimExthet...

Frequency $=2.4$

Main lobe magnitude $=27.3 \mathrm{~dB}$

Main lobe direction $=180.0 \mathrm{deg}$

Angular width $(3 \mathrm{~dB})=7.5 \mathrm{deg}$.

Side lobe level $=-12.1 \mathrm{~dB}$

(a)

\begin{abstract}
Frequency $=2.4$
Main lobe magnitude $=27.3 \mathrm{~dB}$

Main lobe direction $=180.0 \mathrm{deg}$ Angular width $(3 \mathrm{~dB})=3.2 \mathrm{deg}$.

Side lobe level $=-14.1 \mathrm{~dB}$
\end{abstract}

(b)

Figure 7: (a) 2D model of gain radiation pattern of the 128 -antenna array at phi $=0$ deg. (b) $2 \mathrm{D}$ model of gain radiation pattern of the 128 -antenna array at $\mathrm{phi}=90 \mathrm{deg}$.

\section{Conclusion}

In this paper, we have proposed a low-complexity hybrid precoder and a $2 \mathrm{D}$ printed antenna array for $5 \mathrm{G}$ wireless applications. The complexity of hybrid precoder based on $\mathrm{ZF}$ algorithm incurred by direct inversion of large size matrix is greatly reduced by Neumann series approximation. The error performance of this proposed hybrid algorithm is comparable with the fully digital precoding algorithm with reduced complexity. Also, the 128-element antenna array designed using inkjet printing technology shows an excellent gain of $27.34 \mathrm{~dB}$ at the frequency of $2.4 \mathrm{GHz}$ with the return loss of $-15 \mathrm{~dB}$. The proposed antenna array, integrated by 16 subarray modules each comprising $8 \mathrm{Z}$-shaped $\mathrm{CPW}$ 


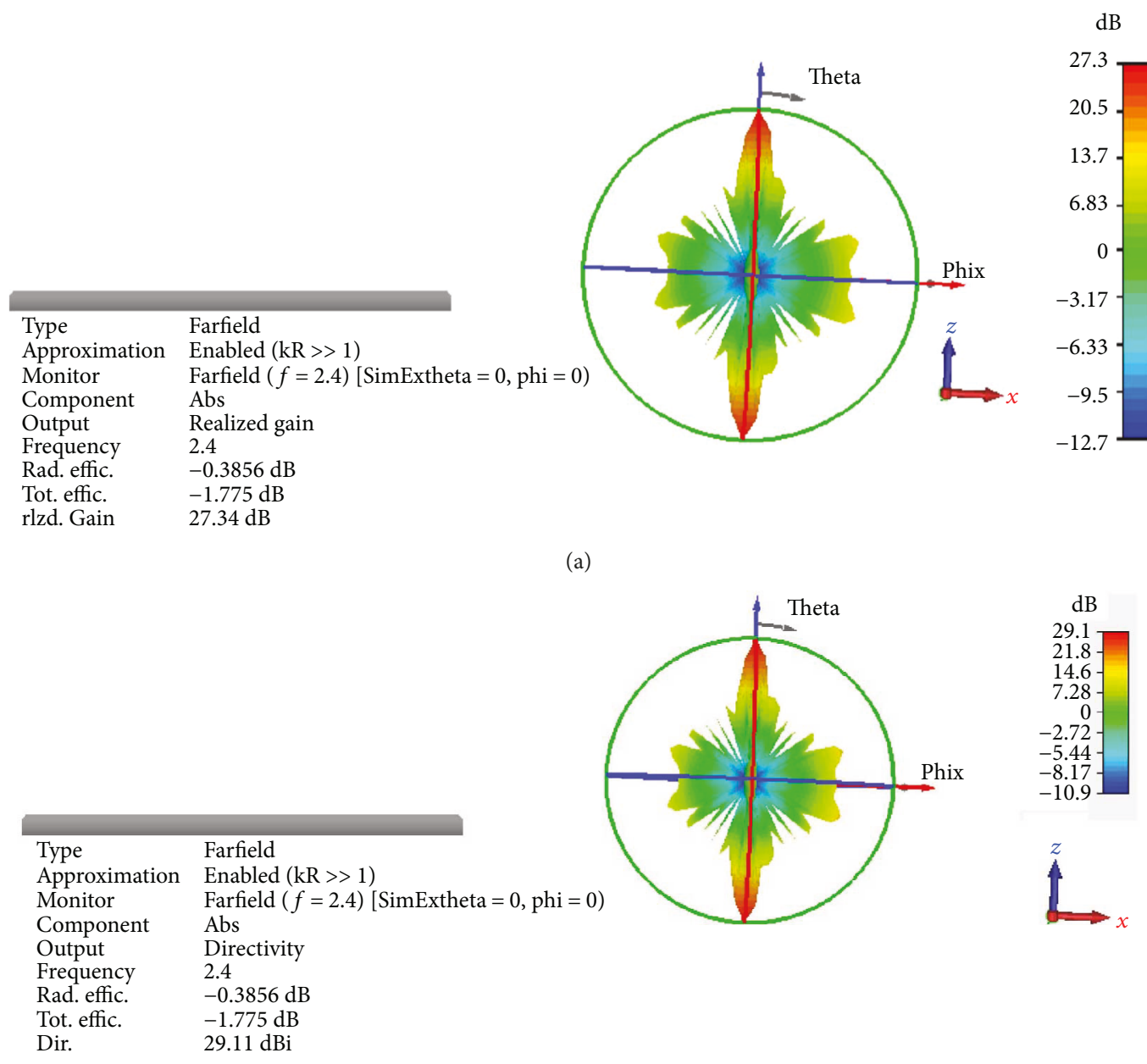

(b)

FIGURE 8: (a) 3D model of the radiation pattern of the gain. (b) 3D model of the radiation pattern of the directivity.

monopole elements, generates multibeam radiation pattern to facilitate the multiuser transmission of $5 \mathrm{G}$ networks. This confirms that the proposed 2D flexible printed antenna array is feasible for future $5 \mathrm{G}$ applications.

\section{Conflicts of Interest}

The authors declare that they have no conflicts of interest.

\section{References}

[1] Huawei Technologies Co., Ltd, New Air Interface and Radio Access Virtualization, White Paper, 2015.

[2] METIS Project, https://www.metis2020.com/.

[3] 5G Forum, http://www.5gforum.org/.

[4] E. Larsson, O. Edfors, F. Tufvesson, and T. Marzetta, "Massive MIMO for next generation wireless systems," IEEE Communications Magazine, vol. 52, no. 2, pp. 186-195, 2014.

[5] T. L. Marzetta, "Noncooperative cellular wireless with unlimited numbers of base station antennas," IEEE Transactions on Wireless Communications, vol. 9, no. 11, pp. 3590-3600, 2010.
[6] H. Huh, G. Caire, H. C. Papadopoulos, and S. A. Ramprashad, "Achieving "massive MIMO" spectral efficiency with a not-solarge number of antennas," IEEE Transactions on Wireless Communications, vol. 11, no. 9, pp. 3226-3239, 2012.

[7] F. Rusek, D. Persson, B. K. Lau, E. G. Larsson, T. L. Marzetta, and F. Tufvesson, "Scaling up MIMO: opportunities and challenges with very large arrays," IEEE Signal Processing Magazine, vol. 30, no. 1, pp. 40-60, 2013.

[8] L. Lu, G. Y. Li, A. Lee Swindlehurst, A. Ashikhmin, and R. Zhang, "An overview of massive MIMO: benefits and challenges," IEEE Journal of Selected Topics in Signal Processing, vol. 8, no. 5, pp. 742-758, 2014.

[9] F. Sohrabi and W. Yu, "Hybrid digital and analog beamforming design for large-scale antenna arrays," IEEE Journal of Selected Topics in Signal Processing, vol. 10, no. 3, pp. 501513, 2016

[10] S. Park, A. Alkhateeb, and R. W. Heath, "Dynamic subarrays for hybrid precoding in wideband mmWave MIMO systems," IEEE Transactions on Wireless Communications, vol. 16, no. 5, pp. 2907-2920, 2017.

[11] M. Kim and Y. H. Lee, "MSE-based hybrid RF/baseband processing for millimeter-wave communication systems in MIMO interference channels," IEEE Transactions on Vehicular Technology, vol. 64, no. 6, pp. 2714-2720, 2015. 
[12] W. Ni and X. Dong, "Hybrid block diagonalization for massive multiuser MIMO systems," IEEE Transactions on Communications, vol. 64, no. 1, pp. 201-211, 2016.

[13] X. Gao, O. Edfors, F. Rusek, and F. Tufvesson, "Linear precoding performance in measured very-large MIMO channels," in 2011 IEEE Vehicular Technology Conference (VTC Fall), San Francisco, CA, USA, September 2011.

[14] H. Prabhu, J. Rodrigues, O. Edfors, and F. Rusek, “Approximative matrix inverse computations for very-large MIMO and applications to linear pre-coding systems," in 2013 IEEE Wireless Communications and Networking Conference (WCNC), pp. 2710-2715, Shanghai, China, April 2013.

[15] A. M. Mansour, N. Shehata, B. M. Hamza, and R. M. Rizk, "Efficient design of flexible and low cost paper-based inkjet printed antenna," International Journal of Antennas and Propagation, vol. 2015, Article ID 845042, 6 pages, 2015.

[16] A. Alkhateeb, G. Leus, and R. W. Heath, "Limited feedback hybrid precoding for multi-user millimeter wave systems," IEEE Transactions on Wireless Communications, vol. 14, no. 11, pp. 6481-6494, 2015.

[17] R. C. de Lamare, "Massive MIMO systems: signal processing challenges and research trends," in Centre for Telecommunications Studies (CETUC), Pontifical Catholic University of Rio de Janeiro, 2013.

[18] L. Hentila, P. Kyosti, M. Kaske, M. Narandzic, and M. Alatossava, MATLAB Implementation of the WINNER Phase II Channel Model Ver 1.1, 2007.

[19] C.-N. Hu, D.-C. Chang, C.-H. Yu, T.-W. Hsaio, and D.-P. Lin, "Millimeter-wave microstrip antenna array design and an adaptive algorithm for future $5 \mathrm{G}$ wireless communication systems," International Journal of Antennas and Propagation, vol. 2016, Article ID 7202143, 10 pages, 2016. 


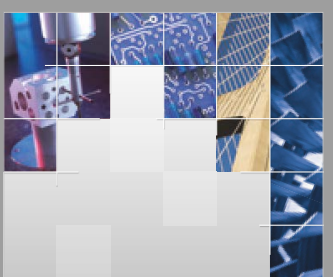

\section{Enfincering}
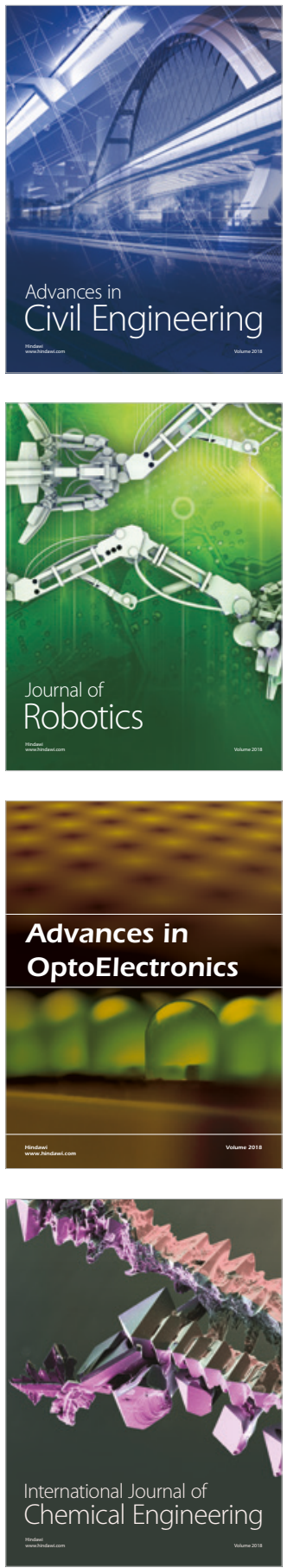

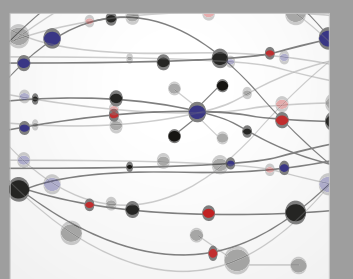

\section{Rotating \\ Machinery}

The Scientific World Journal

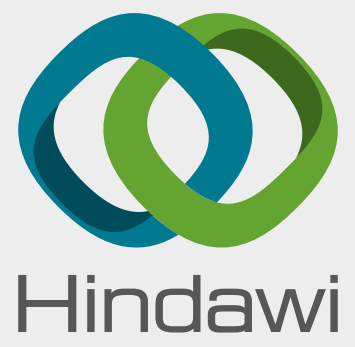

Submit your manuscripts at

www.hindawi.com
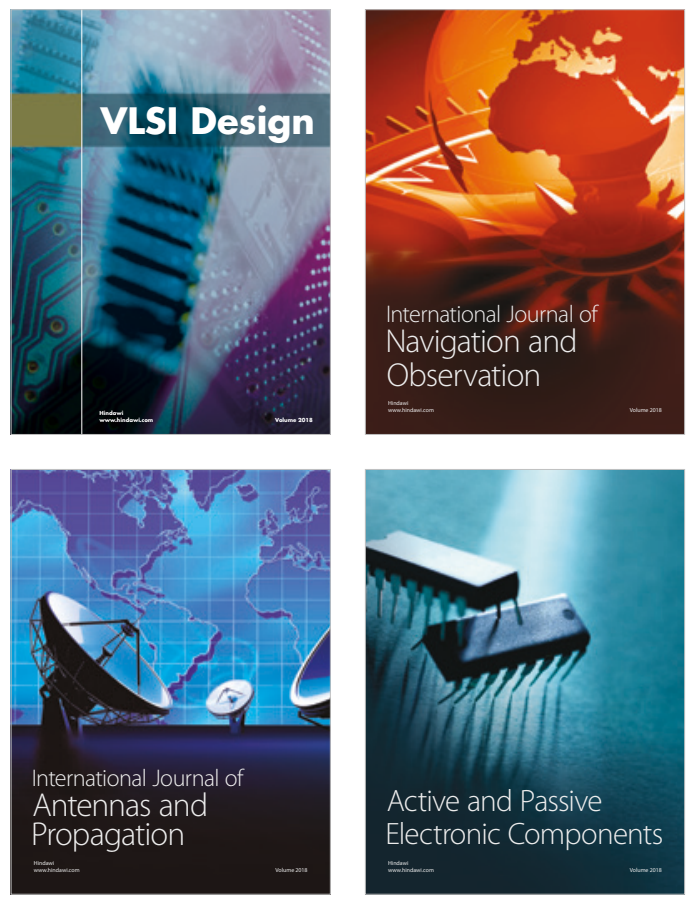
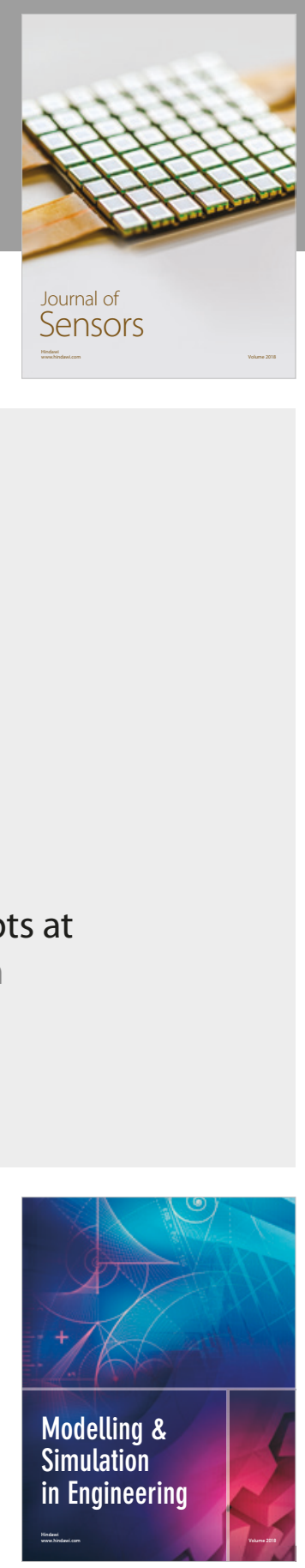

\section{Advances \\ Multimedia}
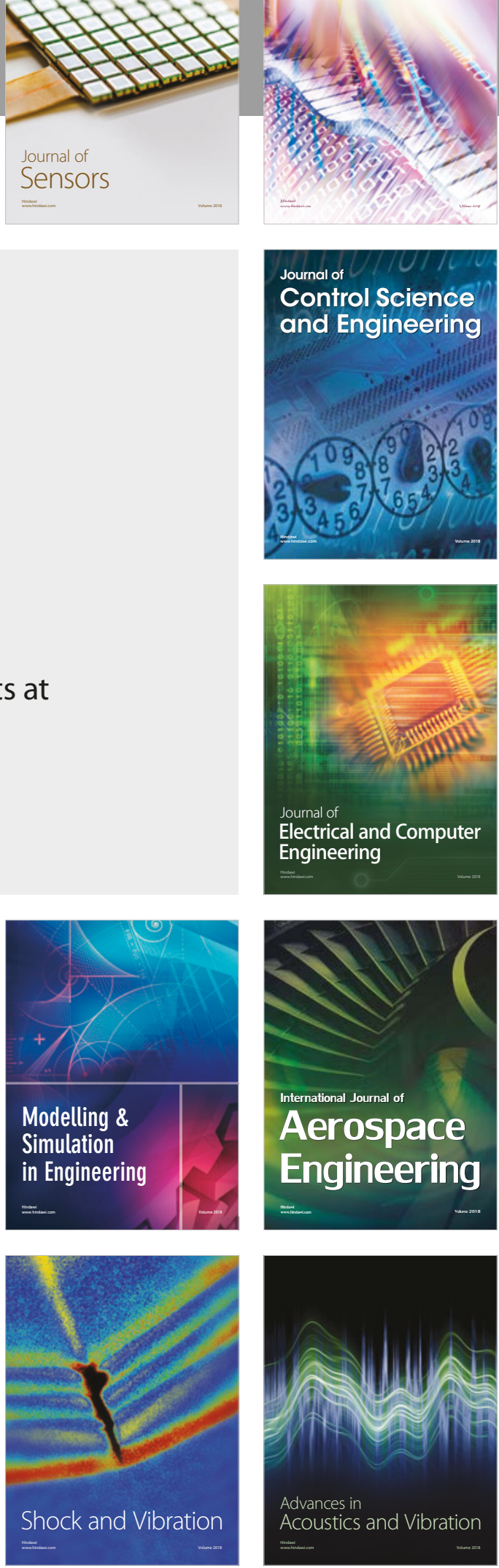